\title{
Medical 3D Printing for the Radiologist, Experience of Marrakech University Hospital Department of Radiology
}

Elhajjami A*, Boutakioute B, Ouali Idrissi M, Idrissi Ganouni N

Radiology Departement, Arrazi Hospital, Mohammed VIth University Hospital Center, Caddi Ayyad University, Marrakech, Morocco

DOI: $10.36347 /$ sjmcr.2021.v09i04.035

| Received: 10.01.2021 | Accepted: 21.01.2021 | Published: 29.04.2021

*Corresponding author: Ayoub Elhajjami

Abstract

Original Research Article

Three-dimensional printing is a manufacturing procedure in which objects are formed by fusing or depositing materials to create a 3D model. Different printers are used for 3D printing. Medical applications for 3D printing are expanding rapidly and are expected to revolutionize health care. 2D radiographic pictures are converted to computerized 3D prints creating anatomical and clinical structures. There are five steps to technically create a 3D model from image and these steps are image acquisition, segmentation, file optimization, choosing materials and printers and print and post processing of 3D medical model. We report the experience of the radiology service at Marrakech university hostpital using a low cost FDM printer. The results of different cases are discussed in this article. Keywords: 3D Printing, Radiologist, Experience.

Copyright (C) 2021 The Author(s): This is an open-access article distributed under the terms of the Creative Commons Attribution 4.0 International License (CC BY-NC 4.0) which permits unrestricted use, distribution, and reproduction in any medium for non-commercial use provided the original author and source are credited.

\section{INTRODUCTION}

Three-dimensional (3D) printing is an assembling technique wherein objects are made by amalgamating or coating materials, for example, plastic, metal, ceramics, powders, fluids, or in any event, living cells - in layers to deliver a 3D object. Some 3D printers are like conventional inkjet printers; nonetheless, the finished result contrasts in that a 3D object is created.

Until now, 3D printing has basically been utilized in designing to make building models. Notwithstanding, ongoing advances in printing materials have now empowered 3D printers to make things that are practically identical with generally fabricated things. Conversely, with customary printers, 3D printing can possibly empower mass customization of products for a huge scope and has significance in medication including ophthalmology. 3D printing has just been demonstrated feasible in a few clinical applications including the production of eyeglasses, custom prosthetic gadgets and dental inserts [1].

Indeed, 3D printing has been utilized in a wide scope of human health care including, however not restricted to the cardiothoracic medical procedure [2], neurosurgery[3], cardiology [2], gastroenterology [4], ophthalmology [5], pulmonology [6], oral and maxillofacial medical procedure [7], urology [8], plastic medical procedure [9], podiatry [10], orthopedic medical procedure [11], radiation oncology [12], transplant medical procedure [12], otolaryngology [13] and vascular medical procedure [14].

Radiologists have seen the development of clinical imaging that takes into consideration 3D printing. Multiplanar imaging with figured tomography (CT), MRI and even ultrasonography offer a large panel of $3 \mathrm{D}$ recreation that improves the assessment of complex anatomy.

In this review, we summarize the $3 \mathrm{D}$ printing workflow in our service, from image acquisition to the generation of a hand-held model, and discuss the applications and the current status and future perspectives.

\section{Material ANd Method}

Method

The pilot study was established over a year (from June 2019 to June 2020). A 3D rapid prototyping laboratory was established in the radiology department, CHU Mohammed VI, Marrakech using low cost 3D printers. 
CT

We used a Siemens Definition As ${ }^{\text {TM }}$ scanner to obtain the images. The slices were $0.63 \mathrm{~mm}$ thick and 0.8 pitch value. The acquisitions were exported in Dicom format.

\section{Materials}

\section{The printers}

- $\quad$ FDM printer: Ender 3 by Creality ${ }^{\mathrm{TM}}$

- Heating plate

- $\quad$ Print size: 220x220x250mm

- Filaments: PLA, ABS

- $\quad$ FDM printer: Volumic stream $20^{\mathrm{TM}}$

- Heating plate

- Print size: 180 x 200 x $240 \mathrm{~mm}$

- Filaments: PLA, ABS

\section{Softwares}

- 3D Slicer: Free image analysis and scientific visualization software, Segmentation and modeling from DICOM image

- Meshmixer: free computer-aided design (CAD) program based on 3D sculpture

- Cura: Free software, which generates a G-code file containing instructions adapted to a specific type of 3D printer

\section{RESULTS}

Case 1: Modeling of a learning support for an anatomical structure: The petrous bone

The specimen has been trimmed to reveal the posterior quadrant of the left side of the skull including the posterior (cerebellar part only) and middle cranial fossa. The model shows the location of the temporal bone and its relationship with the adjoining sphenoid, parietal and occipital bones.

Printing material was gray PLA. The printing time of the model (including support structure) of $63 \mathrm{~g}$ was $5 \mathrm{~h}$ and $43 \mathrm{~min}$ and the dimension obtained was $100 \%$ of the actual size.

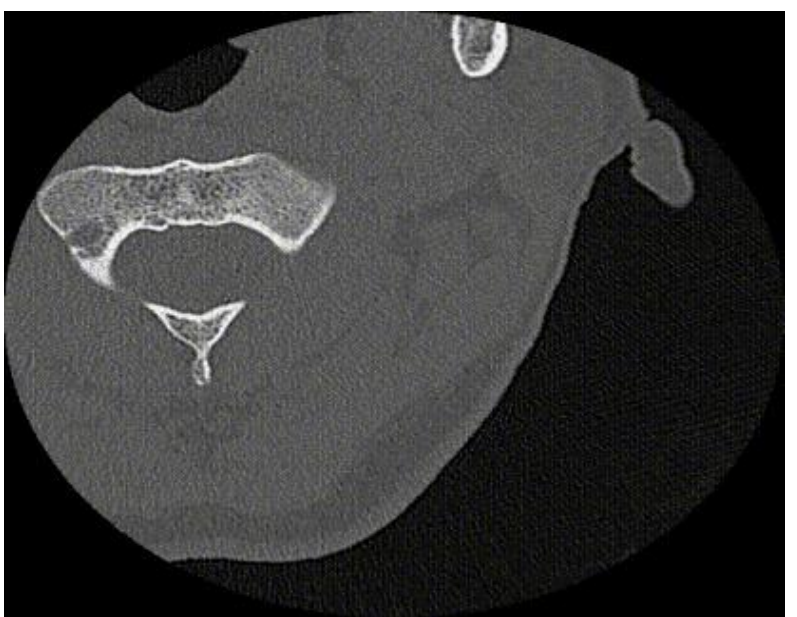

Fig-1: CT scan axial on the crane base

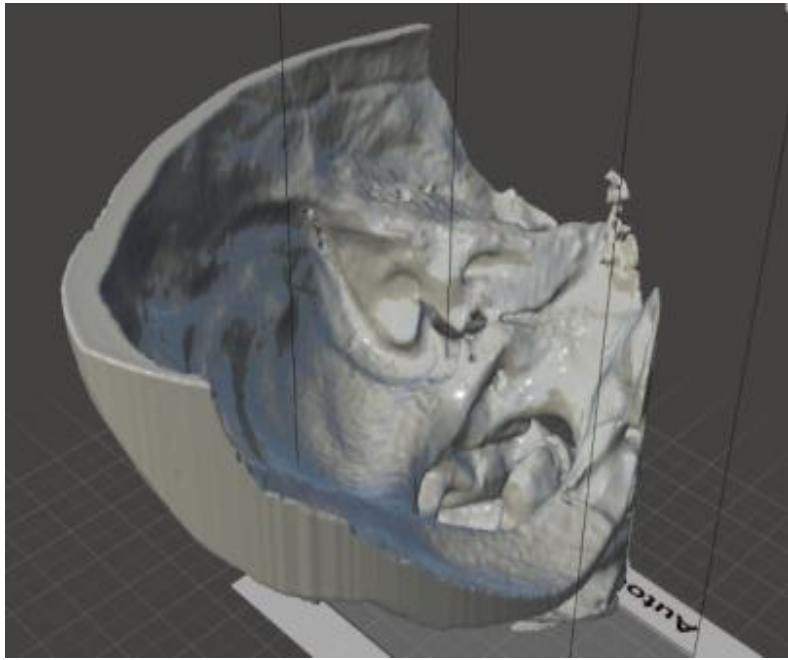

Fig-2: 3D modeling

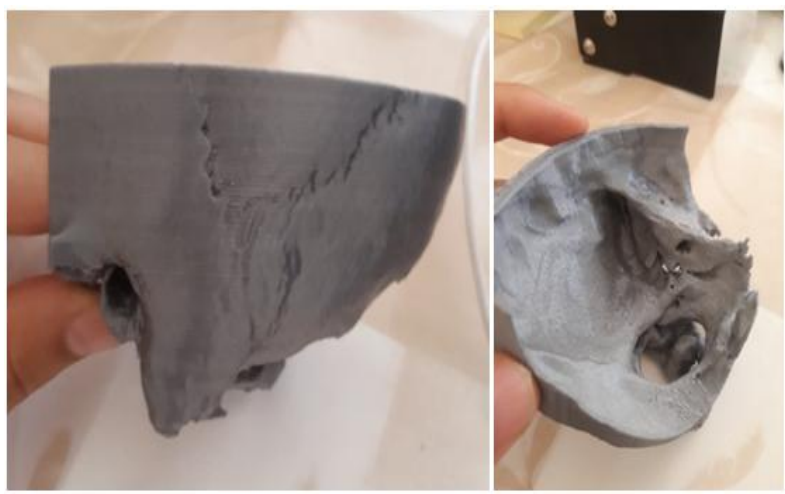

Fig-3: 3D prints

Case 2: Modeling of a support for surgical planning: scoliosis

Spine surgeons dealing with scoliosis are familiar with many of the problems of these complex deformities encountered intra-operatively. Vertebral rotation, absent or dysmorphic pedicles and segmentation anomalies are all components of scoliotic spines and distort the anatomical landmarks for pedicle insertion. Current imaging modalities like CT scans or MRI are often found to be inadequate. This 3D printed anatomical model was used to provide the 'extra' dimension of tactile feedback to the surgeons which can help them anticipate the technical challenges that may be encountered intra-operatively [15].

In our case, 3D reconstruction of a model was done after implementation of an ROI, thresholding and segmentation on a bone window. The model was used for surgical programming for the neurosurgery team at the Mohammed VI university hospital unit and Lyon university hospital for a joint operation.

The material of the model was Gray PLA with $40 \%$. Magnification and the printing time for $60 \mathrm{~g}$ model was 8 h $30 \mathrm{~min}$. 


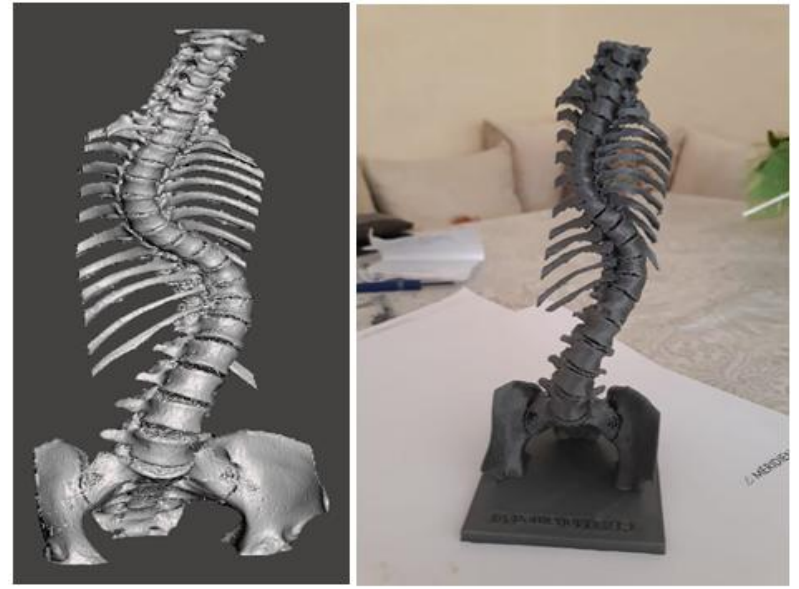

Fig-4: 3D Modeling + 3D print

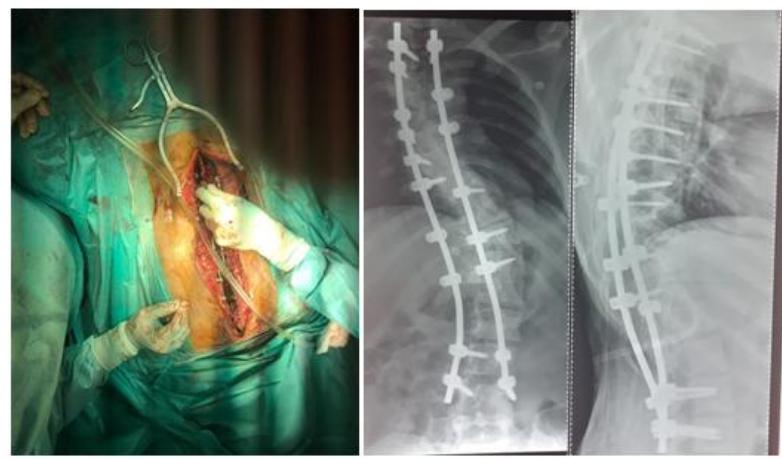

Fig-5: Intraoperative view and post-operative xray control
Case 3: Modeling of a training support for endovascular techniques: Aorta

The 3D-printed model was extracted and printed from anonymized CT and MRI scans. Printing material for model preparation was transparent PLA. Printing time of $110 \mathrm{~g}$ (including support structure) was $12 \mathrm{hrs}$ min with dimension of actual size i.e., $100 \%$ and cropped.

Fluoroscopy has been used as an imaging modality for simulation and allowed an opportunity to rehearse procedures using the medical-grade devices and implants in a safe, zero-risk environment.

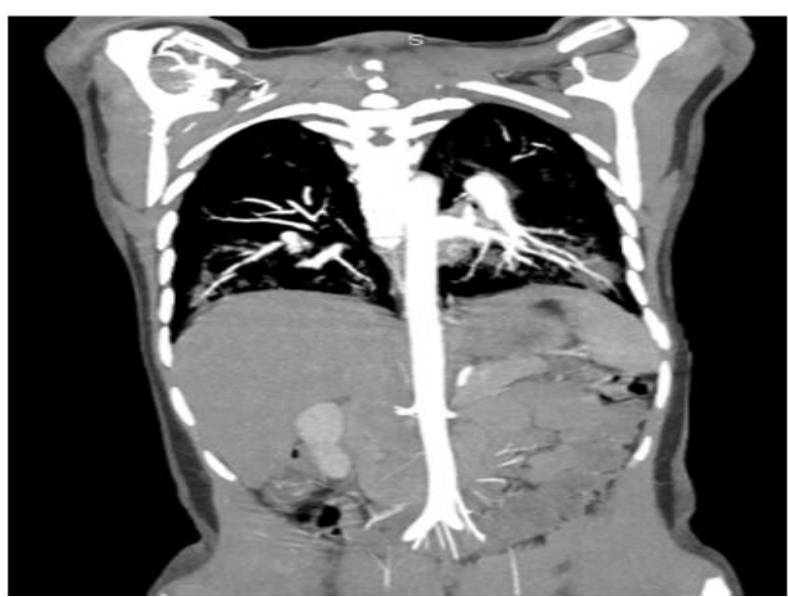

Fig-6: 10 mm MIP coronal CT scan acquisition
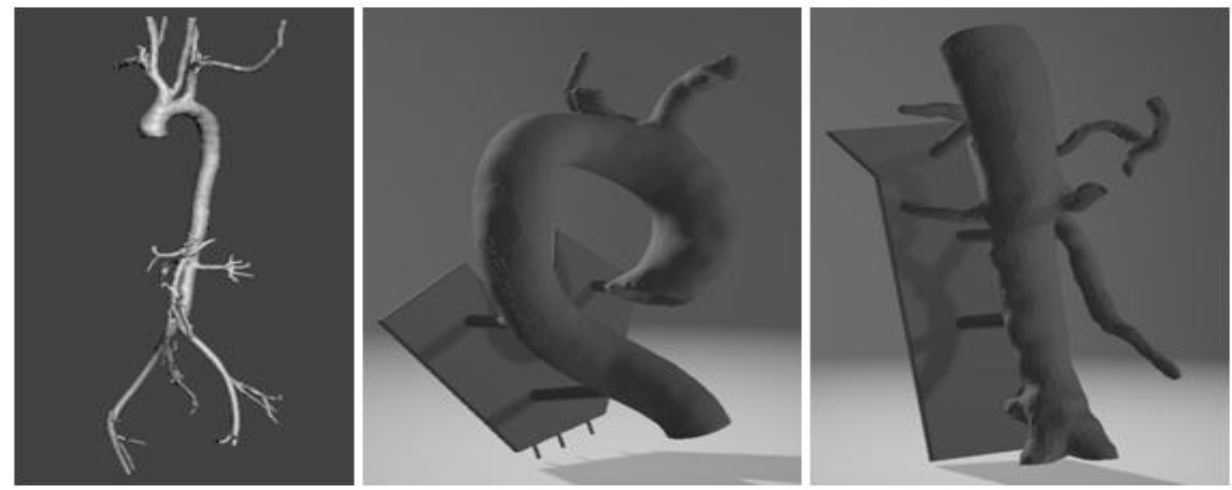

Fig-7: 3D models

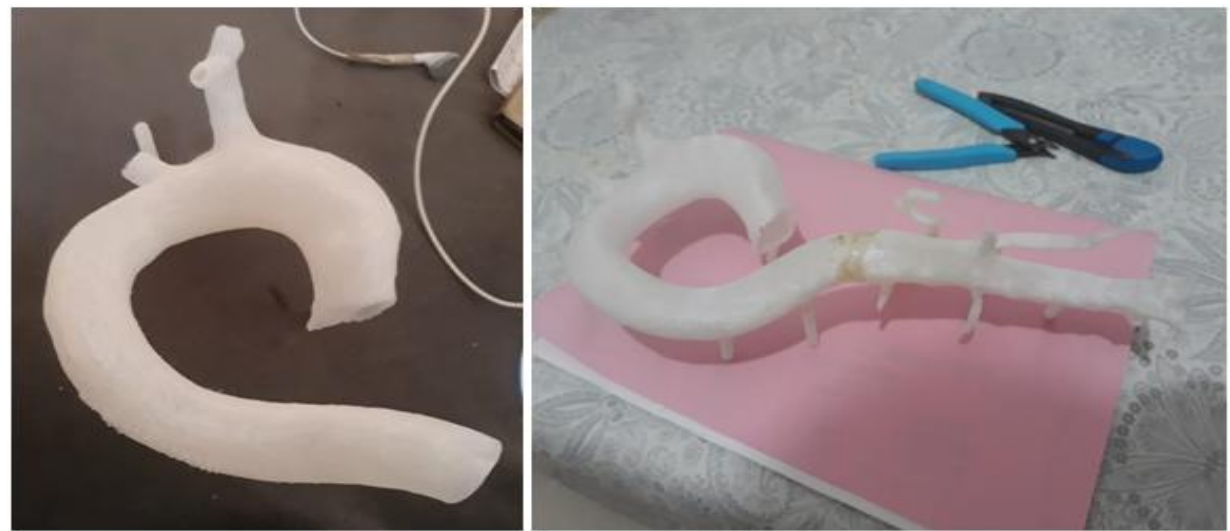

Fig-8: 3D printing in tubular mode 
Case 4: modeling of a support for surgical planning: mandibular trauma

This model was used to plan osteosynthesis and to improve mechanical comprehension of the craniofacial trauma.
The 3D reconstruction of model was performed after implementation of an ROI, thresholding and segmentation on a bone window. Material used was gray PLA. The printing time of model weighing $52 \mathrm{~g}$ with $100 \%$ magnification was $5 \mathrm{~h}$.
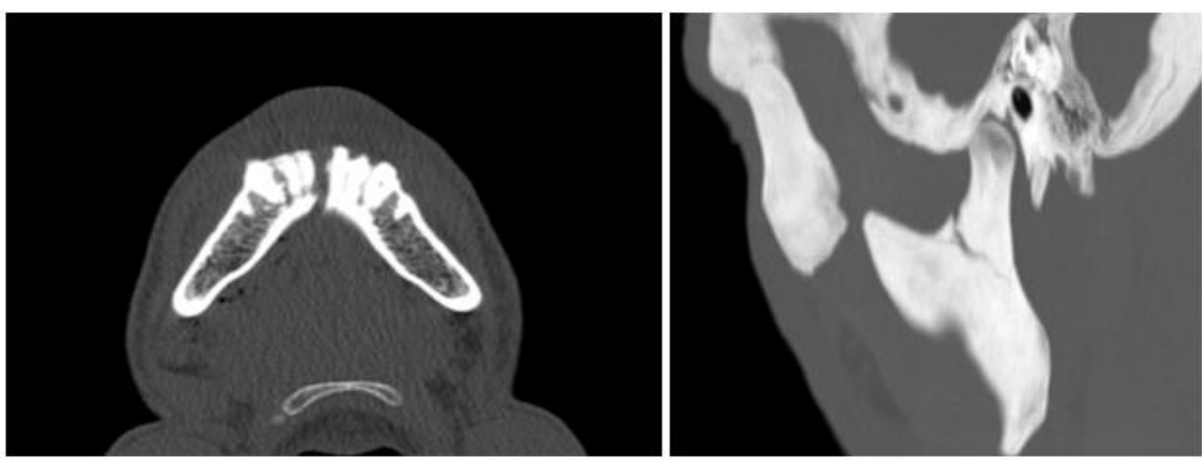

Fig-9: CT scan axial view + sagittal MIP of the mandibule
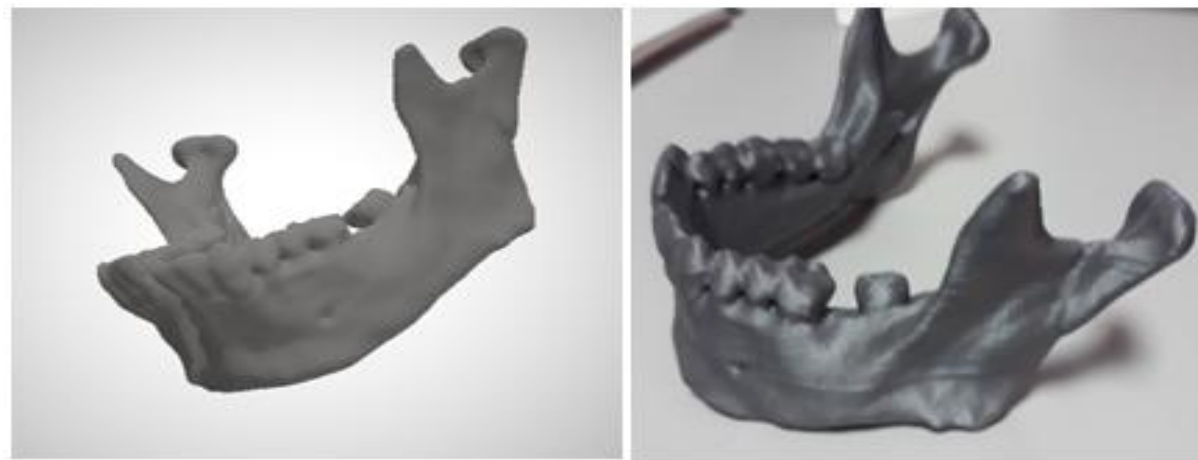

Fig-10: 3d model and 3d print

Case 5: Modeling of a support for surgical planning: trauma of the dorsal spine

This model was used to plan surgical treatment and to tactile feedback to the surgeons to help them anticipate the technical challenges for are complex vertebral fracture that was responsible for me.

Printing material was transparent PLA. The printing time of $36 \mathrm{~g}$ including support structure was $3 \mathrm{~h}$ and $34 \mathrm{~min}$ and the size obtained was actual i.e. $100 \%$ magnification.

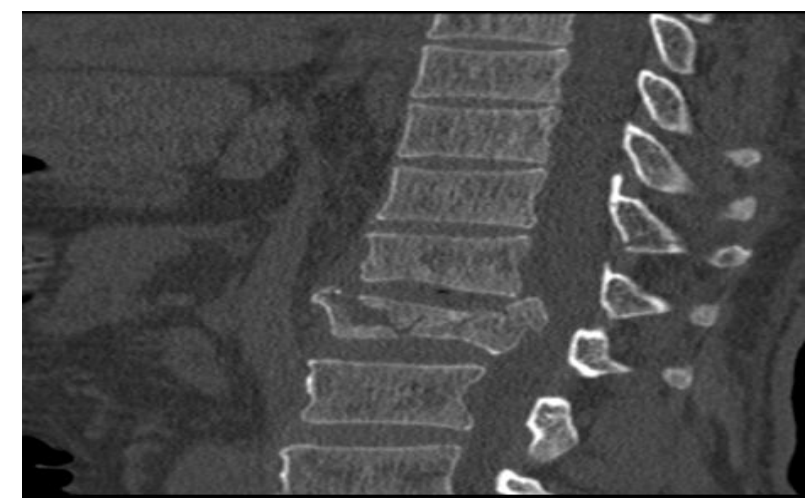

Fig-11: Sagittal CT view on the thoracic spine

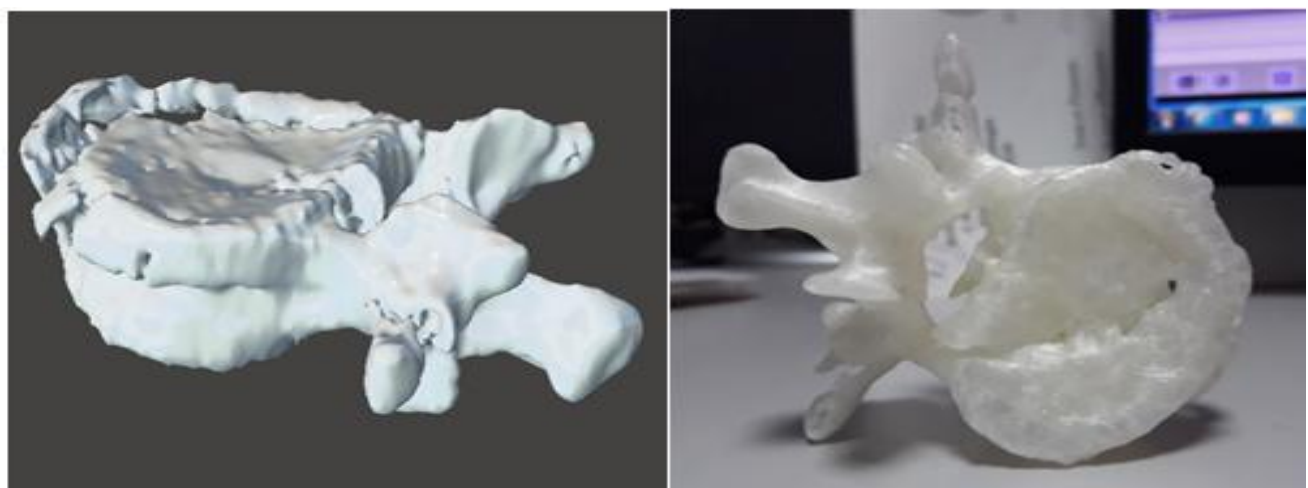

Fig-12: 3D modeling + 3D print 
Case 6: Modeling of a support for surgical planning: complex elbow trauma

This model was used to plan osteosynthesis and to tactile feedback to the surgeons to help them anticipate the technical challenges, complicated joint fractures are challenging to treat

Printing material used was white PLA and the printing weight (including support structure) was $24 \mathrm{~g}$. The printing time was 54 min with reduced size of $25 \%$.
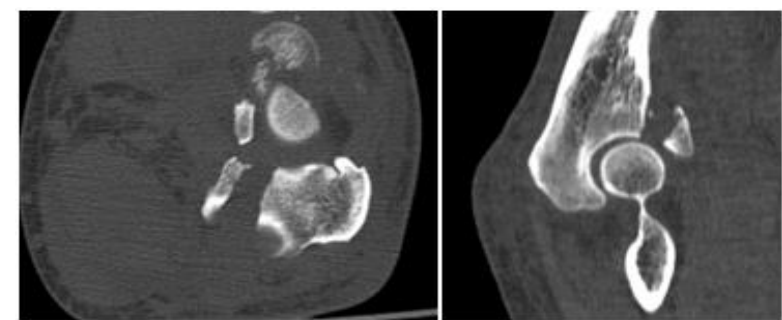

Fig-13: sagittal CT view on the elbow joint

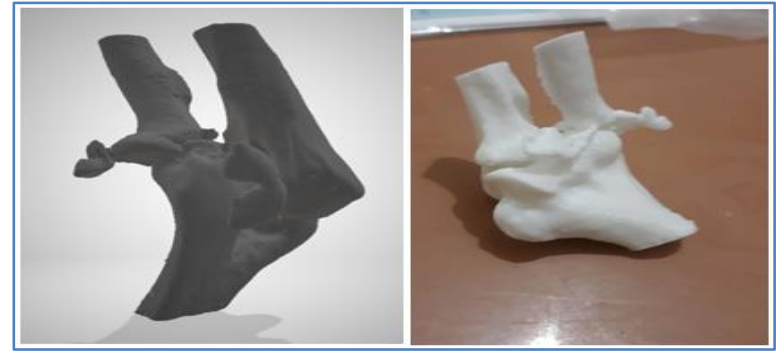

Fig-14: 3d model + 3d print

Case 7: modeling of a support for surgical planning and simulation: mandibular malformation

This patient presents a malar deviation of the left mandibula that was causing pain and discomfort for the patient.

3D reconstruction after implementation of an ROI, thresholding and segmentation on a bone window was performed. The materials used were gray PLA and the printing time was 5 hours for $90 \mathrm{~g}$ weight with $80 \%$ magnification.

The model was printed in two parts two simulate mandibular movement and to plan the best possible osteotomy to provide a result with best mechanics.

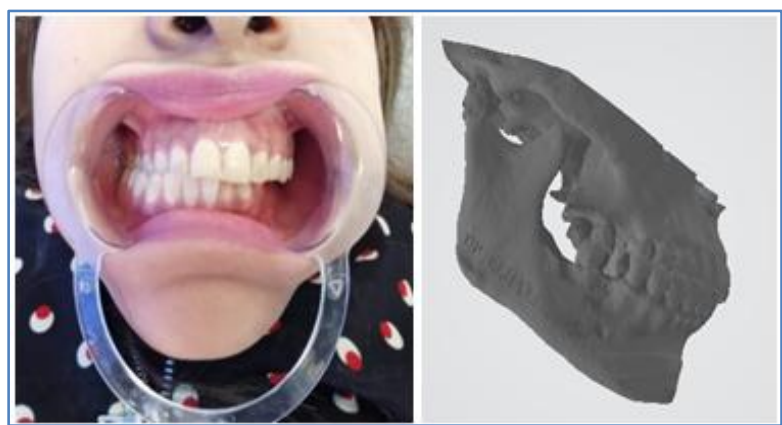

Fig-15: direct examination + 3D modeling

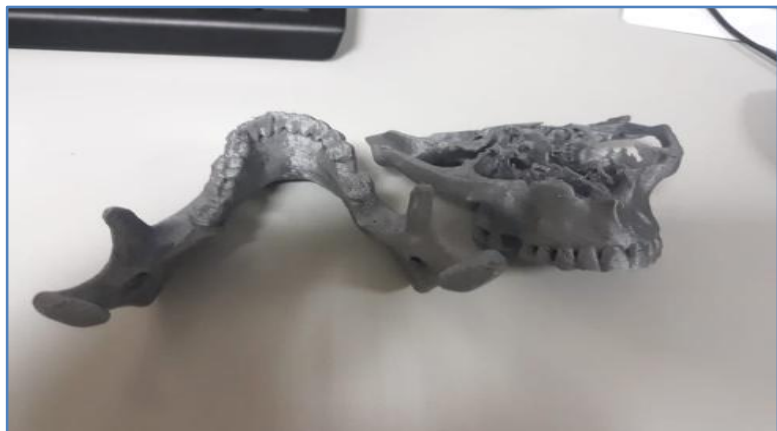

Fig-16: 2-part printing for dynamic visualisation

Case 8: modeling of a support for surgical planning: Aortic aneurysm

This patient presents an aortic root aneurysm 3D print of the aneurysm was realized for surgical planning and localization of the coronary arteries.

3D reconstruction after implementation of an ROI, thresholding and segmentation was performed. The materials used were gray PLA and the printing time was 6 hours for $120 \mathrm{~g}$ weight with $100 \%$ magnification.
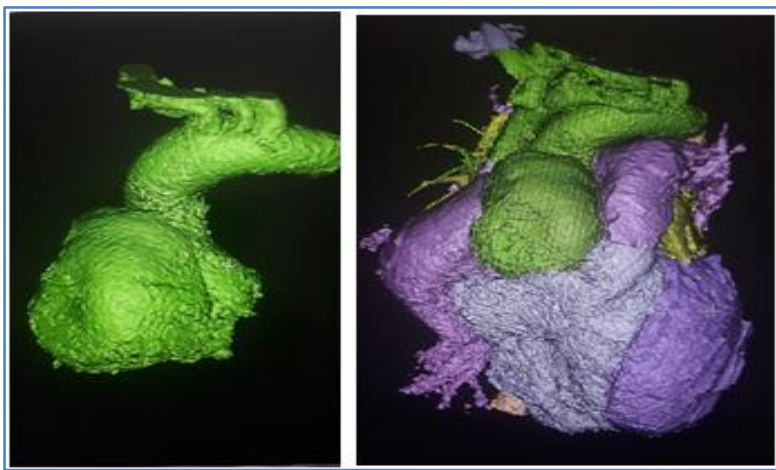

Fig-17: 3d modeling

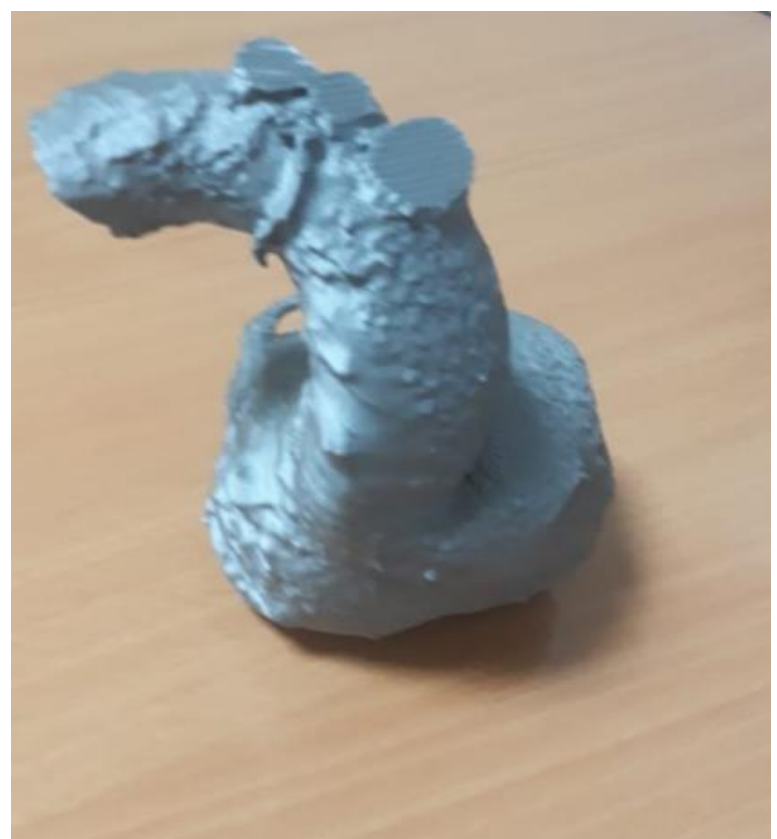

Fig-18: 3d print 


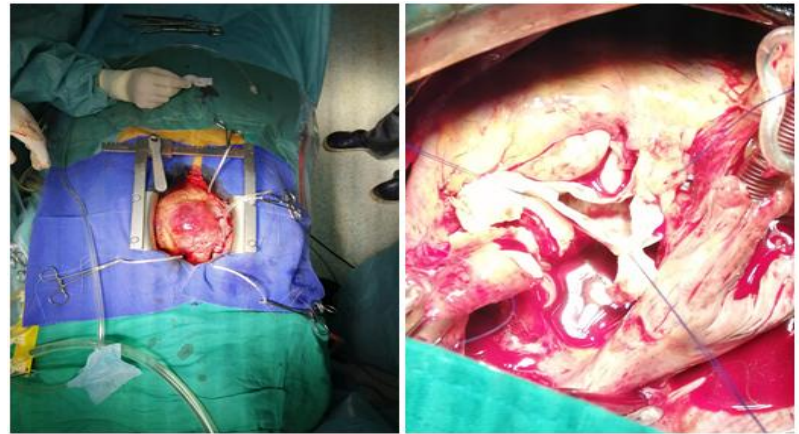

Fig-19: Operative view

\section{DISCUSSION}

There are around two dozen 3D printing forms, which utilize $3 \mathrm{D}$ printer advances and many materials. These innovations can fabricate $3 \mathrm{D}$ objects as characterized in a PC helped structure $(\mathrm{CAD})$ record (Figure 20) [16].

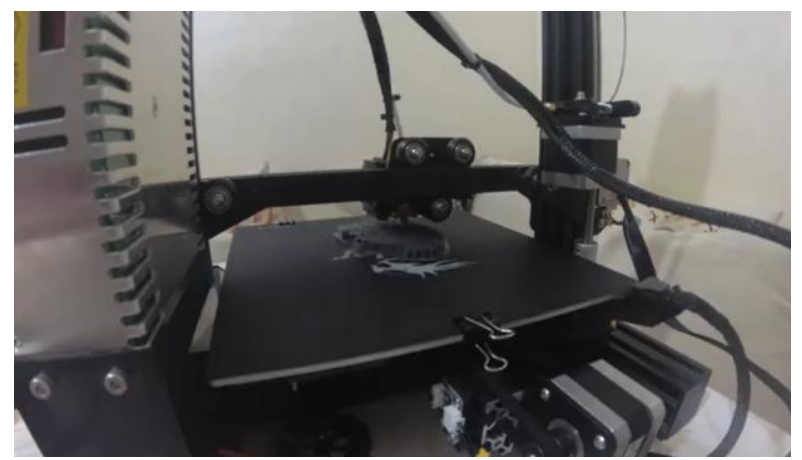

Fig-20: A 3D printer uses instructions in a digital file to create a physical object

In a fundamental arrangement, the 3D printer initially adheres to the guidelines in the CAD document establish the $3 \mathrm{D}$ printed object, moving the printhead along the $\mathrm{x}-\mathrm{y}$ plane. The printer at that point keeps on adhering to the directions, moving the print head along the z-plane to construct the object vertically layer by layer.

Magnetic resonance imaging (MRI), or computerized tomography (CT) exams can be changed over to computerized 3D files, permitting the making of complex anatomical and clinical structures (Figure 2) [7].

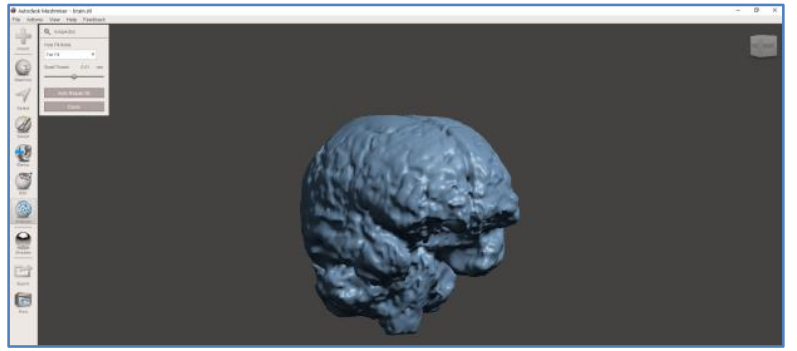

Fig-21: Radiographic images can be converted to 3D print files to create complex, customized anatomical and medical structures
Five technical steps required to finalize a printed model

Five specialized steps are required to make a printed model. They begin by choosing the anatomical territory, the segmentation of the $3 \mathrm{D}$ geometry by the imaging data originating from a CT/MRI exam, the enhancement and correction of the 3D file for physical printing, and choosing the appropriate 3D printer and materials. "slicing" that computerized structure model into a code readable by the 3D printer. The "sliced" structure is then sent to a $3 \mathrm{D}$ printer, which makes the object by beginning at the base layer and building a progression of layers until the item is constructed utilizing the amount of material that is required. An anatomically accurate model made from imaging dataset is finally acquired.

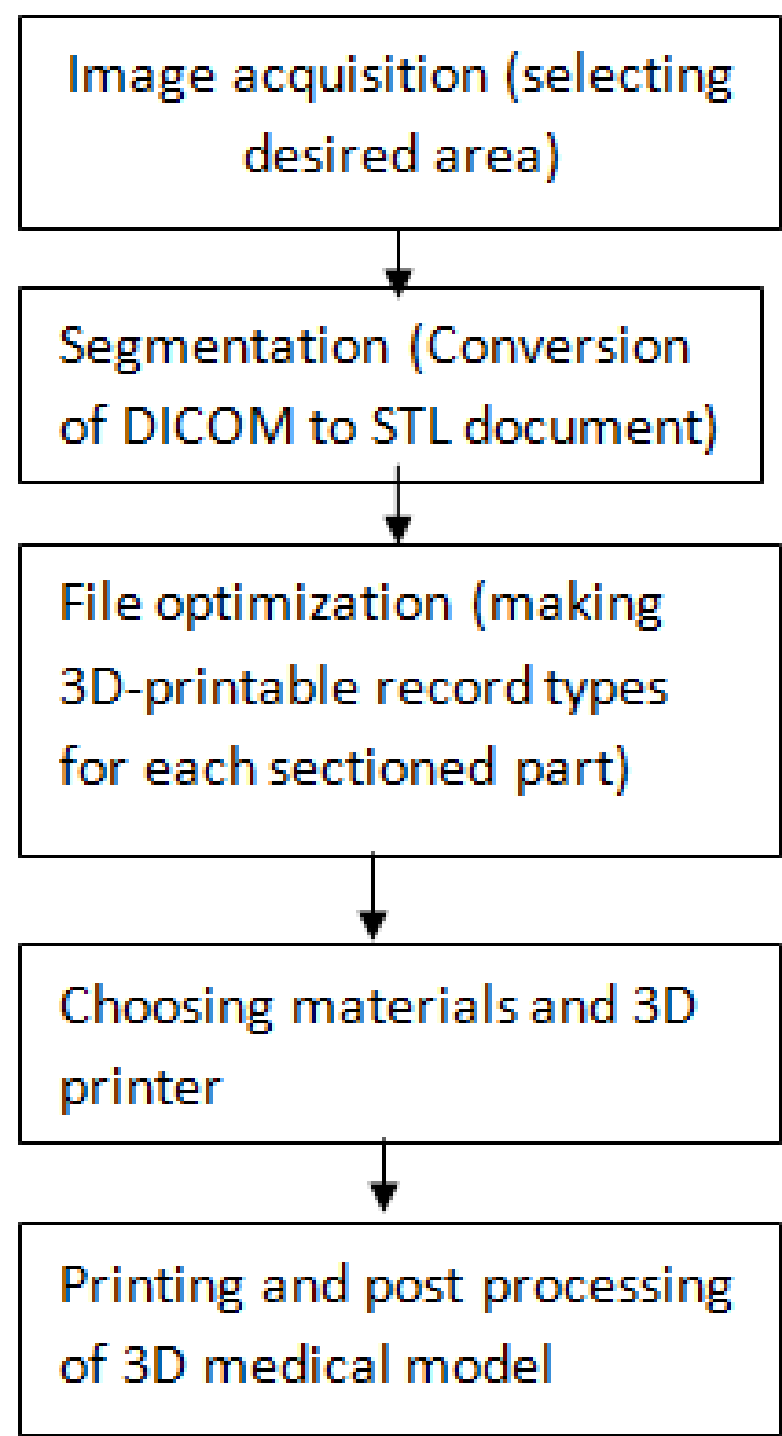

Extraordinary advances have been made in the field of medical 3D printing, including organ models, implants, and $3 \mathrm{~d}$ printed surgery guides.

Despite the fact that the immediate printing of tissues and organs is still in the underlying stages, specialists have using tissues and organs have started to 
contemplate the printing of vessels. Biomaterials utilized in 3D printing include plastic filaments, metals, polymers and ceramics $\left[{ }^{i}\right]$ Recent research on 3D printing for medical purpose can be grouped into these four applications:

1. Preparing organ models to help for surgical and treatment planning, and diagnosis purpose [ $\left.{ }^{\mathrm{ii}}\right]$.

2. Research on customized $3 \mathrm{~d}$ printed nonbioactive implants.

3. research on manufacturing nearby bioactive and biodegradable moulds and surgical guides;

4. research on legitimately printing organs and tissues with complete life capacities [iii]

$3 \mathrm{D}$ printing has been applied in medicine since the early 2000s when the innovation was first used to make dental inserts and custom prosthetics $\left[{ }^{\mathrm{iv}}\right]$. Since, the clinical applications for 3D printing have developed extensively. The current reviews depict the utilization of 3D printing to deliver bones, ears, exoskeletons, eyeglasses, cell cultures, undifferentiated cells, veins, vascular systems, tissues, and organs, just as novel measurement structures and drug delivery devices $\left[{ }^{\mathrm{v}},{ }^{\mathrm{vi}}\right]$. The current clinical employments of $3 \mathrm{D}$ printing can be sorted out into a few general classifications: tissue as well as organ manufacture; making prosthetics, inserts, and anatomical models; and pharmaceutical examination concerning drug revelation, conveyance, and measurement structure $\left[{ }^{\mathrm{vii}}\right]$.

Radiologists have witnessed the evolution of medical imaging that allows for 3D printing. Multiplanar imaging with computed tomography (CT) and magnetic resonance imaging gave rise to $3 \mathrm{D}$ reconstructions that improved the evaluation of complex anatomy [17].

In 2016, the Radiological Society of North America (RSNA) endorsed a proposal to make the Special Interest Gathering on 3D Printing (SIG). This report satisfies two of the first SIG objectives: to give proposals towards predictable and safe creation of 3D printed models got from clinical pictures, and to depict a lot of clinical situations for 3D printing is fitting for the expected utilization of patients' care with those ailments. After the clinical the choice to utilize 3D printing for caring of patients, there are numerous resulting ventures, as investigated in earlier literature $\left[{ }^{\text {viii }},{ }^{\text {ix }},{ }^{\mathrm{x}}\right]$.

\section{CONCLUSION}

$3 \mathrm{D}$ printing has become a useful and potentially revolutionizing tool in the medical area. goes through a series of processes and use different softwares to develop a model. The results of different models show that printer performance, resolution, and available materials play an important role in model preparation. Radiology can be the clinical specialty to take the lead in co ordinating 3D printing services: processing the imaging data, designing 3D models for preoperative planning, and working with surgeons to optimize 3D-printed prostheses and bioactive implants.

\section{REFERENCE}

1. Schubert C, Van Langeveld MC, Donoso LA. Innovations in 3D printing: a 3D overview from optics to organs. British Journal of Ophthalmology. 2014 Feb 1;98(2):159-61.

2. Vukicevic M, Mosadegh B, Min JK, Little SH. Cardiac 3D printing and its future directions. JACC: Cardiovascular Imaging. 2017 Feb 6;10(2):171-84.

3. Randazzo M, Pisapia JM, Singh N, Thawani JP. 3D printing in neurosurgery: a systematic review. Surgical Neurology International. 2016;7(Suppl 33):S801.

4. Jeon H, Kang K, Park SA, Kim WD, Paik SS, Lee SH, Jeong J, Choi D. Generation of multilayered 3D structures of HepG2 cells using a bio-printing technique. Gut and liver. 2017 Jan;11(1):121.

5. Huang W, Zhang X. 3D printing: print the future of ophthalmology. Investigative ophthalmology \& visual science. 2014 Aug 1;55(8):5380-1.

6. Guibert N, Mhanna L, Didier A, Moreno B, Leyx P, Plat G, Mazieres J, Hermant C. Integration of 3D printing and additive manufacturing in the interventional pulmonologist's toolbox. Respiratory medicine. 2018 Jan 1;134:139-42.

7. Saijo H, Igawa K, Kanno Y, Mori Y, Kondo K, Shimizu K, Suzuki S, Chikazu D, Iino M, Anzai M, Sasaki N. Maxillofacial reconstruction using custom-made artificial bones fabricated by inkjet printing technology. Journal of Artificial Organs. 2009 Sep 1;12(3):200-5.

8. Soliman Y, Feibus AH, Baum N. 3D printing and its urologic applications. Reviews in urology. 2015;17(1):20.

9. Chae MP, Rozen WM, McMenamin PG, Findlay MW, Spychal RT, Hunter-Smith DJ. Emerging applications of bedside 3D printing in plastic surgery. Frontiers in surgery. 2015 Jun 16;2:25.

10. Williams C, James A, Chae MP, Hunter-Smith DJ. 3D printing in clinical podiatry: a pilot study and review. Journal of Foot and Ankle Research. 2015 Dec 1;8(S2):O41.

11. Auricchio F, Marconi S. 3D printing: clinical applications in orthopaedics and traumatology. EFORT open reviews. 2016 May;1(5):121-7.

12. Zein NN, Hanouneh IA, Bishop PD, Samaan M, Eghtesad B, Quintini C, Miller C, Yerian L, Klatte R. Three- dimensional print of a liver for preoperative planning in living donor liver transplantation. Liver transplantation. 2013 Dec;19(12):1304-10.

13. Crafts TD, Ellsperman SE, Wannemuehler TJ, Bellicchi TD, Shipchandler TZ, Mantravadi AV. Three-dimensional printing and its applications in otorhinolaryngology-head and neck surgery. 
Otolaryngology-Head and Neck Surgery. 2017 Jun;156(6):999-1010.

14. Hangge P, Pershad Y, Witting AA, Albadawi H, Oklu R. Three-dimensional (3D) printing and its applications for aortic diseases. Cardiovascular diagnosis and therapy. 2018 Apr;8(Suppl 1):S19.

15. Garg B, Mehta N. Current status of 3D printing in spine surgery. Journal of clinical orthopaedics and trauma. 2018 Jul 1;9(3):218-25.
16. Lipson H. New world of 3-D printing offers" completely new ways of thinking": Q\&A with author, engineer, and 3-D printing expert Hod Lipson. IEEE pulse. 2013 Nov 1;4(6):12-4.

17. Ballard DH, Trace AP, Ali S, Hodgdon T, Zygmont ME, DeBenedectis CM, Smith SE, Richardson ML, Patel MJ, Decker SJ, Lenchik L. Clinical applications of 3D printing: primer for radiologists. Academic radiology. 2018 Jan 1;25(1):52-65. 